\title{
The shuriken effect of fertile alpha emitters : a physical process behind findings of chemical toxicity of depleted uranium
}

\section{Florent Pirot}

Unaffiliated researcher

\begin{abstract}
This letter is a contribution on the study of nanoparticles for in situ observation and general understanding of nanoparticle physics. The positing that "chemical" effects from alpha emitters found by researchers actually come from the particular physical effect demonstrated with firmness here is not always possible (as alpha decay also interferes) but a case where this is definitive is given.
\end{abstract}

${ }^{*}$ Correspondence to Author:

Florent Pirot

Unaffiliated researcher

How to cite this article:

Florent Pirot. The shuriken effect of fertile alpha emitters : a physical process behind findings of chemical toxicity of depleted uranium. International Journal of Nanoparticle Research, 2021; 4:15. eSciPub LLC, Houston, TX USA. Website: https://escipub.com/ 


\section{Dear Editors,}

I think that by studying the issue of cell senescence the authors in [1] have forgotten the key factor of alpha decay impacts and associated effects of contamination by alpha emitters. This is of course the explanation for the findings of a pro inflammatory background in [1] that are still interesting, this vocable can relate to such internal contamination even though there are other inflammatory behaviours, deliberate drinking of alcohols of a strong intensity, the quest for extreme sports, the regular exposure to seawater, sunbathing being other examples. Senescence is explained by the pro-inflammatory background. Which is itself encouraged for instance by meat eating, as meat eating encourages for digestive purposes the inhalation of radon, radium fuel fumes etc. as bodily attempt to compensate since our intestines are not made naturally for this (read ${ }^{[3]}$ for more).

I would also like to attract your attention on a 2015 article that shows an application of the antisqueezed principle detailed in ${ }^{[4]}$. Fertile atoms such as $238 \mathrm{U}$ and 232Th happen to have what is called an anti-squeezed shape, which means that particles tend to spread maximally because of the parity of the isotope that is not compatible with a sphere (particles cannot be pressed against each other, so they go outward and an empty space is left in the very core of the atom), they adopt a star-like form (tendencially similar to a sea urchin). Particles (either nano, micro...) constituted mostly of these atoms (the light atoms they come with, i.e. oxygen, fluorine... do not matter really because they are extremely light in comparison with the actinides) keep in aggregate the same structure, in direct application of the principle of the Mengerian sponge. The radium, radon... atoms, particles hit by photons rotate, typically, because of their particular shape (this does not apply to U235, Pu239 and other fissile atoms). This rotation effect creates a damage not related to alpha decay. "Chemical toxicity", the vocabulary used to describe this is incorrect (likewise "cytotoxicity" for instance) - it is a "shuriken effect", not chemical.

A simple detection system relies on observation, simply with eyes, of daylight vs. changes in wavelength combinated with black dots in the middle, to sum up. The black dots show photons that have been mowed down. The photon parts are expelled around with new wavelengths of varying frequencies (but typically outside the visible spectrum), surrounding the black dot. Heavy amounts in e.g. depleted uranium bombs and to a lesser degree - coal plant fumes cause a blackening - strictly proportional with the content of antisqueezed atoms - this is for instance also observable with the Etna (to take a simple example) whose lava and fumes show the strong content in antisqueezed atoms explained by the physics of the formation of the Italian subcontinent (read ${ }^{[5]}$ ). It is reminded that readers should care to take account of all factors. A cloud can be colored in black by non-radioactive oxides from e.g. oil burning, yet in this case, still, black will appear as colour whereas the criteria is the disappearance of light. The usual loss of sun luminosity above big cities due to metropolitan pollution is a typical effect of 226Ra and $228 \mathrm{Ra}$ and their decay products from fuels, for instance (and from coal power, where it is used). When a bomb hits the soil, the effects of the pre-existing radioactivity in the soils have to be taken into account (likewise the military target : depleted uranium armour and ammunition in targeted tanks, for instance). One powerful example is given by the supercriticity in the plutogenic reactor of the Hizbullah militia (fed by depleted uranium harvested in Iraq with magnets...) that was hidden in the harbour of Beirut and that exploded on Aug. 4th 2020 because of a natural phenomenon 
(relativistic neutrons from a space event, that also caused because of fission in some NORM in coal in a mine in Algeria a firedamp explosion on the morning - the plutogenic reactor had a slow rise to supercriticity, as expected with a gas-cooled reactor). The mass of depleted uranium, with a supercriticity in the center of the core, obscures almost totally the flash from the nuclear explosion that happens in that center. Typically, clouds from bombs should be white and rise up quickly. Dark clouds staying near the ground (rising slowly) indicate strong levels of depleted uranium. Images, also, can be manipulated. It is reminded to always watch for signs of image manipulation.

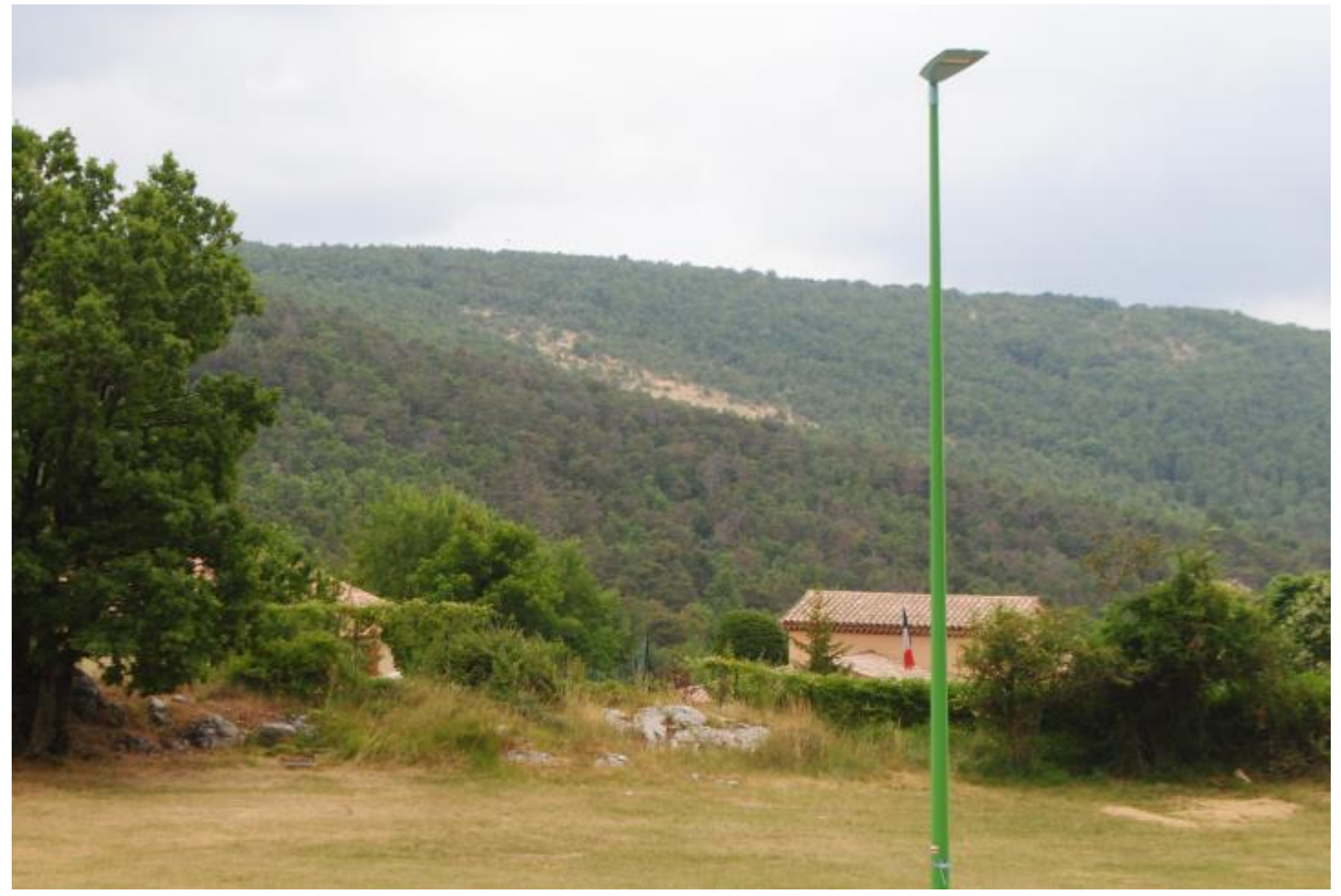

Image description : In the above picture taken in the area of fallout from Canjuers (village of Escragnolles) when the French Army was still firing depleted uranium weapons (picture from July 2019) the combination of luminosity loss and production of new wavelengths on all the background forest is intense - there is a sort of vibration of the atmosphere in general, not only from the new photon wavelengths but from all sorts of waves that are, alike, submitted to the effects of the mowing down at impact onto the anti-squeezed atoms. This conjugates with the fact that the positive polarity of the depleted uranium dust attracts clouds (negatively charged) above.

The interest of the publication ${ }^{[2]}$ is, nevertheless that it helps in casting a new light on the effects of intakes of alpha emitters ; even though "chemical toxicity" is a vocable that has to be discarded since the issue is unrelated with the electronic bonding of the atoms and linked, instead to physical properties of these atoms, these results demonstrate how sunlight, for instance, 
conjugates with internal contamination with alpha emitters to increase their dangerosity for health, creating the inflammatory effects that we go through everyday. Toxicology in general as a science should in the future focus on chemical warfare agents such as sarin, VX, mustard gas..., instead of molecules used in industrial processes - where co-contamination with alpha emitters is usually rife and always forgotten.

\section{References}

[1] Maduro, Ana T. BSca,b; Luís, Carla MScb,c; Soares, Raquel PhDb,c,*Ageing, cellular senescence and the impact of diet: an overview, Porto Biomedical Journal: January/February 2021 Volume 6 - Issue 1 - p e120 doi: 10.1097/j.pbj. 0000000000000120

[2] Wilson, J., Zuniga, M. C., Yazzie, F., and Stearns, D. M. (2015) Synergistic cytotoxicity and DNA strand breaks in cells and plasmid DNA exposed to uranyl acetate and ultraviolet radiation, J. Appl. Toxicol., 35; page 338 - 349, doi: 10.1002/jat. 3015.

[3] Pirot F. "Spitting as traditional medicine" - why

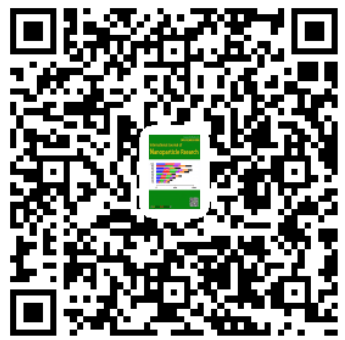
alpha-emitting nanoparticulates make spitting an unfortunate but significant contribution to health. International Journal of Traditional and Complementary Medicine 2021; 6:33. DOI: 10.28933/ijtcm-2021-01-2306

[4] Pirot F, Fissile vs. Fertile Atoms Renamed Squeezed State vs. Anti-squeezed States, Why Uranium, Thorium Etc are Invisible in Star Spectrometry Data, and a Return on Human Behaviour, Cultural Insecurity Defined as Anti-squeezed Behaviour, Linked to Brain Trauma, International Journal of Physics. 2020, 8(3), 108-113. DOI: 10.12691/ijp-8-3-4

[5] Pirot F, Fermionic Condensation Explains the Formation of Subcontinents and Small Volcanic Islands around Them - General Geophysical Rules. International Journal of Physics. 2021; 9(1):42-47. doi: 10.12691/ijp-9-1-5 